\title{
Pattern formation and evolution near autocatalytic reaction fronts in a narrow vertical slab
}

\author{
Jie Huang and Boyd F. Edwards \\ Department of Physics, West Virginia University, P.O. Box 6315, Morgantown, West Virginia 26506-6315
}

(Received 20 November 1995)

\begin{abstract}
Linear analysis and nonlinear numerical simulations of autocatalytic reaction fronts ascending in narrow vertically unbounded slabs describe the growth, development, and annihilation of fingers in the front, the dynamics of edge suppression, and a secondary transition to a two-roll state above the onset of convection. The pattern formation and evolution of the reaction fronts are determined by the horizontal aspect ratio $\Gamma=b / a$ and the dimensionless driving parameter $S=\delta g a^{3} / \nu D_{C}$, which involve the gap thickness $a$, the slab width $b$, the fractional density difference $\delta$ between the unreacted and reacted solutions, the gravitational acceleration $g$, the kinematic viscosity $\nu$, and the catalyst molecular diffusivity $D_{C}$. The reaction fronts satisfy a chemical reaction-diffusion equation and two-dimensional Navier-Stokes equations describing the average Poiseuille velocity in the vertical plane perpendicular to the gap direction. The wavelength of maximum growth rate reaches a minimum value at $a \approx 1 \mathrm{~mm}$. [S1063-651X(96)13109-3]
\end{abstract}

PACS number(s): 47.20.Bp, 47.70.Fw, 03.40.Gc, 82.20.Mj

\section{INTRODUCTION}

Autocatalytic reaction-diffusion fronts [1] in aqueous solution serve as a rich arena for the study of pattern formation and evolution, combining chemical reaction-diffusion phenomena with hydrodynamic flow [2]. Iodate-arsenous-acid reaction fronts consume unreacted solution to produce a reacted solution of lower mass density. Buoyancy therefore renders ascending fronts potentially unstable to convection [2]. A slab with parallel vertical walls separated by a small gap provides an ideal geometry to investigate pattern formation in chemical waves. The purpose of this paper is to investigate the theory of pattern formation and evolution for ascending autocatalytic reaction fronts in such a geometry.

An autocatalytic front of iodide in iodate-arsenous-acid solution serves as a unique system because of its simplicity. The reaction front can be accurately described by the oxidation of iodide by iodate and the reduction of iodine by arsenous acid; the iodide is generated autocatalytically at the front, and diffuses ahead of the front. A simple autocatalytic reaction-diffusion equation, derived from the chemical reaction-rate equations and the catalyst diffusion equation, governs the evolution of the front in the absence of convection. It successfully describes the one-dimensional propagation of the front in a vertical capillary tube [3].

An isothermal hydrodynamic stability theory of convection near autocatalytic reaction fronts [2] treats the thin reaction front as a moving surface which consumes unreacted fluid of uniform mass density $\rho_{u}$ to produce reacted fluid of lower uniform density $\rho_{r}$, thereby relegating all chemical reactions to the surface. The relative strength of buoyancy is measured by a dimensionless driving parameter (Ref. [4]),

$$
S=\frac{\delta g a^{3}}{\nu D_{C}},
$$

where $\delta=\left(\rho_{u}-\rho_{r}\right) / \rho_{r}$ is the fractional density difference between unreacted and reacted fluids, $g$ the acceleration of gravity, $a$ the gap thickness, $\nu$ the kinematic viscosity, and $D_{C}$ the molecular diffusivity of the catalyst. In this "thin- front" approximation, the reaction-diffusion equation reduces to a simple "eikonal" relation [5] between the front velocity and the front curvature. In a reference frame stationary with respect to the fluid, the eikonal relation gives the normal component of velocity of the reaction front as $c=c_{0}+D_{C} K$, where $c_{0}$ is the flat front speed and $K$ is the front curvature. Here $K$ is measured as positive when the center of curvature is in the unreacted fluid. This curvature correction tends to lower peaks and raise valleys in the front surface, thus flattening the front. For ascending fronts, buoyancy competes with this curvature effect, and tends to destabilize a flat front.

In this paper, we consider a narrow slab that is unbounded in the vertical direction $z$, with gap $a$ and width $b>a$, and with no-slip boundaries at $x= \pm a / 2$ and $y= \pm b / 2$. For a sufficiently small gap, laminar viscous flows are two dimensional, being restricted to the plane parallel to the slab. The two components of velocity in this plane, which must vanish at the no-slip walls, have a well-characterized quadratic Poiseuille dependence on the coordinate $x$ normal to the walls [6]. Averaging the Navier-Stokes equations over this coordinate (Sec. II) yields a set of equations involving the two components of averaged velocity, which depend on $y, z$, and the time $t$. These two-dimensional equations lend themselves to linear and nonlinear analyses, and reduce to Darcy's law for steady irrotational flow. In Sec. III, based on these twodimensional equations and the correspondingly averaged eikonal equation, we summarize the results of a linear stability analysis for the onset of convection for autocatalytic reaction fronts in a horizontally unbounded slab with $b \rightarrow \infty$, and compare them with three-dimensional results obtained previously. We also investigate the effect of the sidewalls at $y= \pm b / 2$, for finite $b$. In Sec. IV, we discuss a numerical simulation of front evolution above the onset of convection for finite $b$ by coupling the two-dimensional equations with the reaction-diffusion equation, which is more convenient for numerical simulation than the eikonal equation. In Sec. V, we present the results of the numerical simulation, discuss pattern formation and evolution near the autocatalytic reaction fronts, and draw conclusions. 


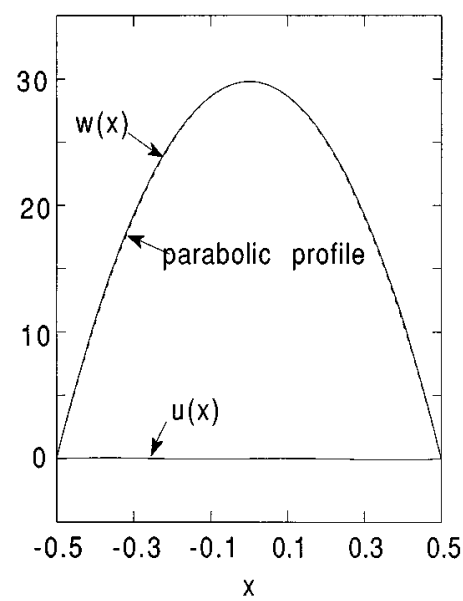

FIG. 1. Even-parity convective velocity field [6] for $q_{c}=0.5 \mathrm{vs}$ horizontal coordinate $x$ perpendicular to a vertical slab. The component of the velocity field perpendicular to the slab is represented by $u(x)$, and the vertical component by $w(x)$, with $|u| \approx 0.005|w|$. The dashed curve is the parabolic profile in Eq. (4) chosen to have the same maximum magnitude as $w(x)$.

\section{EQUATIONS OF MOTION FOR A NARROW SLAB}

The Navier-Stokes equations for incompressible fluids,

$$
\begin{gathered}
\frac{\partial \mathbf{v}}{\partial t}+(\mathbf{v} \cdot \nabla) \mathbf{v}=\mathbf{f}-\frac{1}{\rho} \nabla P+\nu \nabla^{2} \mathbf{v}, \\
\nabla \cdot \mathbf{v}=\mathbf{0},
\end{gathered}
$$

involve the fluid velocity $\mathbf{v}$, the external force per unit volume $\mathbf{f}$, the pressure $P$, the mass density $\rho$, and the kinematic viscosity $\nu$. Fully three-dimensional analytical solutions obtained previously for the onset of convection for autocatalytic reaction fronts in a vertical slab (with $b \rightarrow \infty$, Ref. [6]) reveal that, when $a$ is small, the magnitude of the $x$ component $u$ of the velocity, which is normal to the slab plane, is much smaller than the magnitudes of the $y$ and $z$ components $v$ and $w(|u| \approx 0.005|w|)$. These solutions also show that $v$ and $w$ have parabolic Poiseuille profiles in the $x$ direction (see Fig. 1) satisfying no-slip boundary conditions. Accordingly, we ignore $u$ and demand such profiles on $v$ and $w$ by writing

$$
\mathbf{v}(x, y, z, t)=\frac{6}{a^{2}}\left(\frac{a^{2}}{4}-x^{2}\right) \mathbf{V}(y, z, t) .
$$

Here, $\mathbf{V}=\overrightarrow{v \mathbf{y}}+\overrightarrow{w \mathbf{z}}$ is the fluid velocity $\mathbf{v}$ averaged over $-a / 2 \leqslant x \leqslant a / 2$. Substituting Eq. (4) into Eqs. (2) and (3) and averaging yield

$$
\begin{aligned}
\frac{\partial \mathbf{V}}{\partial t}+\frac{6}{5}(\mathbf{V} \cdot \bar{\nabla}) \mathbf{V}= & \overline{\mathbf{f}}-\frac{1}{\rho} \bar{\nabla} \bar{P}+\nu \bar{\nabla}^{2} \mathbf{V}-\frac{12 \nu}{a^{2}} \mathbf{V}, \\
& \bar{\nabla} \cdot \mathbf{V}=\mathbf{0},
\end{aligned}
$$

where $\bar{\nabla}=(\partial / \partial y) \hat{\mathbf{y}}+(\partial / \partial z) \hat{\mathbf{z}}$, and $\overline{\mathbf{f}}$ and $\bar{P}$ are averaged quantities. Equations (5) and (6) describe the averaged nonlinear two-dimensional fluid motion between two parallel planes, based on the Poiseuille velocity profile in Eq. (4). The last two terms in Eq. (5) come from the average of $\nu \nabla^{2} \mathbf{v}=\nu\left(\partial^{2} / \partial x^{2}+\bar{\nabla}^{2}\right) \mathbf{v}$.

It is appropriate to give some historical perspective on Eq. (5). In the absence of external forces, we can ignore the acceleration term on the left side to obtain Darcy's law [7]

$$
\mathbf{V}(y, z)=-\frac{a^{2}}{12 \rho \nu} \bar{\nabla} \bar{P}
$$

for steady irrotational flows. Darcy's law can readily be modify to incorporate external forces $\overline{\mathbf{f}}$ necessary for the study of the displacement of one fluid by another in a HeleShaw geometry $(a \rightarrow 0)$ [8]. Replacing the term $\nu \nabla^{2} \mathbf{v}$ in Eq. (2) by $-12 \nu \mathbf{V} / a^{2}$, that is, ignoring the $\bar{\nabla}^{2}$ term and the factor $6 / 5$ in Eq. (5), yields a set of equations used to study the stability of a binary fluid mixture in the Hele-Shaw geometry [9]. This replacement reduces the order of the differential equations, making it impossible to invoke no-slip boundary conditions at $y= \pm b / 2$. To overcome this problem, Brinkman [10] suggested that the $\bar{\nabla}^{2}$ term be included. Accordingly replacing the Laplacian in Eq. (2) by $\nabla^{2}=\bar{\nabla}^{2}-12 / a^{2}$ yields Eq. (5), except for the factor $6 / 5$. The $\bar{\nabla}^{2}$ term predicts important finite-gap corrections in double diffusive systems [11].

At an interface between these two viscous fluids with unit normal $\hat{n}$, the fluid velocity $\mathbf{v}$ and the normal stress $n_{j} T_{i j}$ for $(i, j=x, y, x)$ must be continuous [12]. Here the threedimensional stress tensor $T_{i j}=P \delta_{i j}+T_{i j}^{v}$ consists of a diagonal component $P \delta_{i j}$ and a viscous component $T_{i j}^{v}=-\mu\left(\partial v_{i} / \partial x_{j}+\partial v_{j} / \partial x_{i}\right)$. Averaging as before yields a two-dimensional stress tensor $\bar{T}_{i j}=\bar{P} \delta_{i j}+\bar{T}_{i j}^{V}$ with $T_{i j}^{V}=-\mu\left(\partial V_{i} / \partial x_{j}+\partial V_{j} / \partial x_{i}\right)$ for $(i, j=y, z)$. Therefore, we demand continuous $\mathbf{V}$ and continuous $n_{j} \bar{T}_{i j}$ at the interface between the two fluids.

The hydrodynamic stability theory of convection near autocatalytic reaction fronts [2] treats the thin reaction front as a moving surface which consumes unreacted fluid to produce reacted fluid. By defining a time-dependent reaction-front height $z=h(x, y, t)$ and a unit vector $\hat{\mathbf{n}}$ pointing normal to the front into the unreacted fluid, we can write the normal front velocity relative to the moving fluid as $c=\hat{\mathbf{n}} \cdot \hat{\mathbf{z}} \partial h / \partial t-\left.\hat{\mathbf{n}} \cdot \mathbf{v}\right|_{z=h}$. This involves the normal fluid velocity at the front $\left.\hat{\mathbf{n}} \cdot \mathbf{v}\right|_{z=h}$ and the normal front velocity $\hat{\mathbf{n}} \cdot \hat{\mathbf{z}}(\partial h / \partial t)$ in the laboratory frame. When the gap $a$ is sufficiently small, both this equation and the eikonal relation, $c=c_{0}+D_{C} K$, are replaced by the averaged equations, respectively. The no-chemical-flow boundary condition $\partial h / \partial x=0$ at $x= \pm a / 2$ demands vanishing averaged curvature perpendicular to the plane, which therefore makes no contribution to the eikonal relation. Accordingly,

$$
\begin{aligned}
& \overline{\mathbf{n}} \cdot\left(\hat{\mathbf{z}} \frac{\partial \bar{h}}{\partial t}-\left.\mathbf{V}\right|_{z=\bar{h}}\right)=c_{0}+D_{C} \bar{K} . \\
& \text { III. ONSET OF CONVECTION } \\
& \text { FOR } \boldsymbol{b} \rightarrow \infty \text { AND FOR FINITE } \boldsymbol{b}
\end{aligned}
$$

\section{ONSET OF CONVECTION FOR $b \rightarrow \infty$ AND FOR FINITE $b$}

We now summarize the results of a linear stability analysis of autocatalytic reaction fronts in a narrow vertical slab 
that is unbounded in the vertical direction and in one horizontal direction $(b \rightarrow \infty)$. The associated horizontal translational invariance allows unrestricted perturbation wavelengths in this direction. As usual [2], we employ the Oberbeck-Boussinesq approximation [13] by neglecting the small density difference between the reacted and unreacted fluids except in the large gravity term. Equations (5), (6), and (8) govern the evolution of the averaged fluid velocity $\mathbf{V}(y, z, t)$ and the averaged reaction front height $z=\bar{h}(y, t)$. These equations allow us to apply standard linear stability techniques $[6,2]$ to study the fully time-dependent equations. Whereas Ref. [6] discusses three-dimensional solutions for the marginal state only, we are here able to obtain solutions of the two-dimensional time-dependent equations. These solutions allow us to study the maximum growth rate, which is of particular interest experimentally.

This linear stability analysis yields the growth rate $\sigma$ for a perturbation wave number $q$ and a driving parameter $S$,

$$
\begin{gathered}
S q\left[2 q+\left(k_{+}-k_{-}\right)\right]-2\left(q^{2}+\sigma \nu / D_{c}\right)\left(k_{+}-k_{-}\right) \\
\times\left[2 q^{2}+12+\sigma+q\left(k_{+}-k_{-}\right)\right]=0,
\end{gathered}
$$

where $k_{ \pm}=-3 V_{0} / 5 \pm \sqrt{q^{2}+12+\sigma+9 V_{0}^{2} / 25}$. Here $\sigma$ and $q$ are dimensionless quantities measured in units of $\nu / a^{2}$ and $1 / a$, respectively. For iodate-arsenous-acid reaction fronts, $V_{0}=c_{0} a / \nu \approx 0.03$ for $a \approx 1 \mathrm{~mm}, \quad$ so $9 V_{0}^{2} / 25 \sim 3.24$ $\times 10^{-4} \ll 12$. Neglecting this term in $k_{ \pm}$and simplifying Eq. (9) yields

$$
S q-2\left(q^{2}+\sigma \nu / D_{c}\right)\left(q^{2}+12+\sigma+q \sqrt{q^{2}+12+\sigma}\right)=0 .
$$

For the marginal state, the perturbations neither grow or decay with time, so that $\sigma=0$. Equation (10) therefore becomes

$$
S=2 q_{c}\left(q_{c}^{2}+12+q_{c} \sqrt{q_{c}^{2}+12}\right),
$$

which relates the marginal wave number $q_{c}$ to the driving parameter $S$. Figure 2 compares this condition with the exact three-dimensional marginal condition for the onset of convection for autocatalytic reaction fronts in a vertical slab with arbitrary gap $a$ [6]. These both agree as $q_{c} \rightarrow 0$ with the "Hele-Shaw" limit $S \rightarrow 24 q_{c}$ obtained from Darcy's law, agree that $S \rightarrow 4 q_{c}^{3}$ as $q_{c} \rightarrow \infty$, and differ by at most $5.2 \%$ at $q_{c} \approx 6$. In our previous work [6], we obtained a useful approximate analytical marginal condition relating $S$ and $q_{c}$,

$$
S=2 q_{c}\left(q_{c}^{2}+12+q_{c} \sqrt{q_{c}^{2}+18}\right),
$$

which agrees with the exact three-dimensional condition to within $2.5 \%$ over the whole range of $S$.

For iodate-arsenous-acid reaction fronts, $\nu=9.2 \times 10^{-3}$ $\mathrm{cm}^{2} / \mathrm{s}$ and $D_{c}=2.0 \times 10^{-5} \mathrm{~cm}^{2} / \mathrm{s}$. Solving Eq. (10) numerically yields the growth rate $\sigma$ as a function of the wave number $q$ for various values of $S$ (see Fig. 3). As indicated in Fig. 3, the larger the driving parameter $S$, the larger the growth rate $\sigma$ for a given perturbation wave number $q$. Taking the derivative of Eq. (10) with respect to $q$ and setting $d \sigma / d q=0$ yield

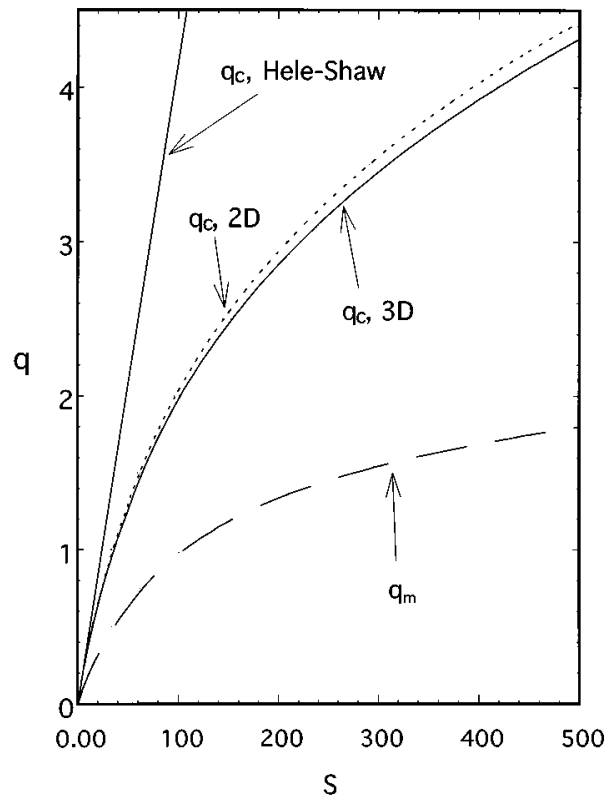

FIG. 2. Convective stability diagram for flat ascending reaction fronts in a vertical slab with unbounded width $b \rightarrow \infty$. The threedimensional (3D) trace for $q_{c}$ represents the marginal stability obtained by solving the three-dimensional Navier-Stokes equations [6], the 2D trace that obtained by the two-dimensional equations (5) and (6), and the Hele-Shaw trace that obtained by Darcy's law, Eq. (7). For a particular value of the driving parameter $S$, the band $0<q<q_{c}$ of perturbation wave numbers is unstable to convection. The wave number $q_{m}$ with the maximum linear growth rate for given $S$ is obtained from the two-dimensional equations.

$$
\begin{gathered}
\sigma_{m}=\frac{D_{C} q_{m}^{2}}{\nu}\left[\frac{2\left(q_{m}^{2}+12+\sigma_{m}\right)}{12+\sigma_{m}-q_{m} \sqrt{q_{m}^{2}+12+\sigma_{m}}}-1\right], \\
S=\frac{4 q_{m}\left(q_{m}^{2}+12+\sigma_{m}\right)\left(q_{m}^{2}+12+\sigma_{m}+q_{m} \sqrt{q_{m}^{2}+12+\sigma_{m}}\right)}{12+\sigma_{m}-q_{m} \sqrt{q_{m}^{2}+12+\sigma_{m}}} .
\end{gathered}
$$

The maximum growth rate $\sigma_{m}$ occurs at the perturbation wave number $q_{m}$, which is uniquely determined by the driving parameter $S$ (Fig. 2). Numerical solutions of Eqs. (13) and (14) reveal that $\sigma_{m}<2 \times 10^{-2}$ for $S<300$. Accordingly ignoring $\sigma_{m}$ on the right sides of Eqs. (13) and (14) yields the approximate relations

$$
\begin{gathered}
\sigma_{m}=\frac{D_{C} q_{m}^{2}}{\nu}\left[\frac{2\left(q_{m}^{2}+12\right)}{12-q_{m} \sqrt{q_{m}^{2}+12}}-1\right], \\
S=\frac{4 q_{m}\left(q_{m}^{2}+12\right)\left(q_{m}^{2}+12+q_{m} \sqrt{q_{m}^{2}+12}\right)}{12-q_{m} \sqrt{q_{m}^{2}+12}} .
\end{gathered}
$$

These relations agree with the numerical solutions of Eqs. (13) and (14) to within $0.02 \%$ for both $\sigma_{m}$ and $S$ at the typical value $q_{m}=1.0$, and have the advantage of giving $\sigma_{m}$ and $S$ directly from $q_{m}$.

For the iodate-arsenous-acid reaction fronts, Eq. (1) indicates that the slab gap thickness $a$ determines the driving 


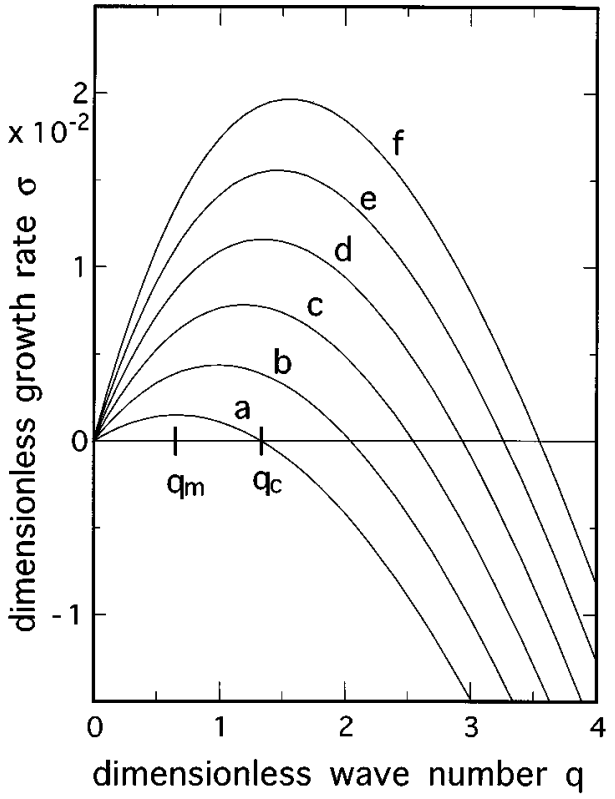

FIG. 3. Dimensionless linear growth rate $\sigma$ vs wave number $q$ for $S=50(\operatorname{trace} a), 100(\operatorname{trace} b), 150(\operatorname{trace} c), 200($ trace $d), 250$ (trace $e$ ), and 300 (trace $f$ ). The critical wave number $q_{c}$, the growth rate $\sigma$, and the wave number $q_{m}$ with maximum growth rate $\sigma_{m}$ all increase with increasing $S$. The maximum growth rate is less than $2 \times 10^{-2}$ for $S<300$. The quantities $q_{c}$ and $q_{m}$ are labeled for $S=50(\operatorname{trace} a)$.

parameter $S$, which determines the wave number $q_{m}$ of maximum growth rate (Fig. 2). Thus the maximum growth wavelength $\lambda_{m}=2 \pi a / q_{m}$ is determined by the gap $a$. Solving Eq. (9) numerically yields Fig. 4, which reveals the existence of a critical slab gap $a_{c}$. The wavelength $\lambda_{m}$ decreases to a minimum value as $a$ increases to the critical value $a_{c}$, increases with $a$ as $a>a_{c}$, and approaches the maximum growth wavelength $\lambda_{m}=15.0374\left(\nu^{2} / \delta g\right)^{1 / 3}$ for a laterally unbounded system [2] as $a \rightarrow \infty$. The wavelength $\lambda_{m}$ behaves quite differently than the cutoff wavelength $\lambda_{c}$, which de-

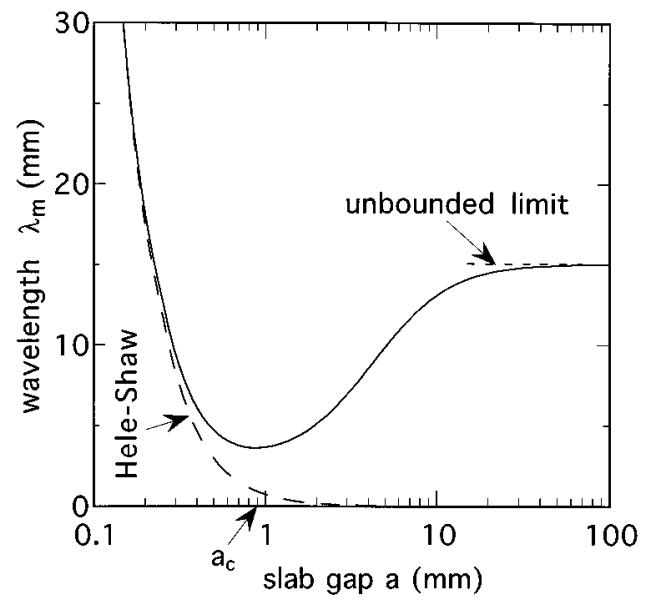

FIG. 4. The wavelength $\lambda_{m}$ of maximum growth rate vs slab gap $a$ for the iodate-arsenous-acid system (solid trace), including the Hele-Shaw and laterally unbounded limits. The wavelength $\lambda_{m}$ reaches its minimum value as $a=a_{c}$.

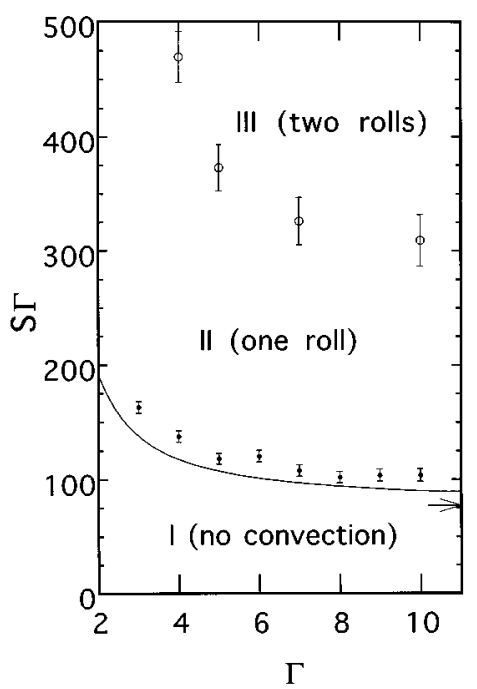

FIG. 5. Stability diagram for ascending fronts in a vertical slab with finite width $b$. The solid line represents the predicted marginal state, and the arrow indicates its asymptotic limit $S \Gamma=76.1$ for $\Gamma \rightarrow \infty$, which is close to the asymptotic limit $24 \pi$ for laterally unbounded slab. The filled circles with error bars represent the marginal state obtained from our numerical simulations. The front is flat and convectionless in region I; one convective roll appears in region II. The open circles with error bars locate the transition from the one-roll state to a symmetric two-roll state.

creases monotonically with $a$, and approaches the cutoff wavelength $\lambda_{c}=2 \pi\left(4 \nu D_{C} / \delta g\right)^{1 / 3}$ for the laterally unbounded system as $a \rightarrow \infty$ (see Fig. 3 in Ref. [6]). Solving Eq. (9) numerically with finite $V_{0}$ yields values of $q_{m}$ and $\sigma_{m}$ that agree with Eqs. (13) and (14) to within $0.03 \%$ at typical values of $a=0.2,1,10$, and $100 \mathrm{~mm}$.

Finite width $b$ eliminates horizontal translational symmetry and requires rigid boundary conditions at the sidewalls,

$$
\left.\mathbf{V}\right|_{y= \pm \Gamma / 2}=\mathbf{0}
$$

where $\Gamma=b / a$, the aspect ratio of the slab. We use the algorithm of Vasques, Edwards, and Wilder [4] to obtain the marginal condition for $\mathcal{S}_{c}$ and $\Gamma$ as plotted in Fig. 5 (solid trace).

Comparing with results for $b \rightarrow \infty$ yields a deeper understanding of the marginal condition for finite $b$ in Fig. 5. The minimum wavelength $\lambda_{c}=2 \pi a / q_{c}$ (measured in conventional units) necessary for convection decreases with increasing driving parameter $S$ (Fig. 2). Since each wavelength $\lambda$ of the convection pattern embodies two rolls, a single roll in a finite- $b$ cell corresponds to the maximum allowable wavelength $\lambda=2 b$ for such a cell. Thus, $\lambda_{c}=2 b$ embodies an approximate marginal condition for the onset of convection for finite $b$. This condition relates the corresponding marginal aspect ratio $\Gamma=\pi / q_{c}$ to $S$ through Eq. (11). This relation underestimates the marginal condition in Fig. 5 by about $10 \%$.

\section{NUMERICAL SIMULATION}

The reaction kinetics of a propagating front of iodide in an iodate-arsenous-acid solution can be accurately described 
by the oxidation of iodate by iodate and the reduction of iodine by arsenous acid [3]. The iodide is generated autocatalytically at the reaction front and diffuses ahead of the front, catalyzing the reaction. In the absence of convection, the coupling of the corresponding chemical reaction rate equations with the catalyst diffusion equation governs the spatiotemporal evolution of the iodide concentration and the propagation speed of the iodide front. The simple autocatalytic reaction-diffusion equation describing a onedimensional iodide front in an iodate-arsenous-acid reaction with arsenous acid in stoichiometric excess $\left(\left[\mathrm{H}_{3} \mathrm{AsO}_{3}\right]_{0}>3\left[\mathrm{IO}_{3}{ }^{-}\right]_{0}\right)$ has been investigated [3],

$$
\frac{\partial C}{\partial t}=D_{C} \frac{\partial^{2} C}{\partial z^{2}}-\alpha C\left(C-C_{2}\right)\left(C-C_{3}\right),
$$

where $C=\left[\mathrm{I}^{-}\right]$denotes the iodide concentration, $C_{2}=\left[\mathrm{IO}_{3}{ }^{-}\right]_{0}=5.0 \times 10^{-3} \mathrm{M}$ the initial iodate concentration, $C_{3}=-k_{a} / k_{b}=-1.03 \times 10^{-5} M, \quad$ and $\quad \alpha=k_{b}\left[\mathrm{H}^{+}\right]^{2}=3.45$ $\times 10^{4} \mathrm{M}^{-2} / \mathrm{s}$. The reaction rate constants are $k_{a}=4.50$ $\times 10^{3} \mathrm{M}^{-3} / \mathrm{s}$ and $k_{b}=3.45 \times 10^{8} \mathrm{M}^{-4} / \mathrm{s}$. Equation (18) resolves the reaction front, which was treated previously as infinitesimally thin using the eikonal equation, Eq. (8). Instead of the time-dependent front height $\bar{h}$, Eq. (18) naturally introduces the scalar variable $C$, which avoids the discontinuity at the front and is consequently more accurate and convenient for numerical simulations. Previous calculations demonstrate that the two approaches agree for the thin fronts of interest [14]. Equation (18) has a steady state solution,

$$
C(\xi)=\frac{C_{2}}{1+\left(C_{2} / C_{0}-1\right) e^{k \xi}},
$$

where $\quad \xi=z-v_{0} t, \quad k=\sqrt{\alpha / 2 D_{C}} C_{2}, \quad$ and $\quad v_{0}$ $=\sqrt{2 \alpha D_{C}}\left(C_{2} / 2-C_{3}\right)$, the propagation speed of the front. For the values given above, $v_{0}=2.95 \times 10^{-3} \mathrm{~cm} / \mathrm{s}$. The parameter $C_{0}$ is the initial iodide concentration satisfying $C_{0} \ll C_{2}$ (Ref. [3]).

Including hydrodynamics, the governing equations for the evolution of the iodide front in iodate-arsenous-acid solution are

$$
\begin{gathered}
\frac{\partial C}{\partial t}+\mathbf{V} \cdot \bar{\nabla} C=D_{C} \bar{\nabla}^{2} C-\alpha C\left(C-C_{2}\right)\left(C-C_{3}\right), \\
\rho(C)=\rho\left(C_{2}\right)\left[1-\beta\left(C-C_{2}\right)\right],
\end{gathered}
$$

and Eqs. (5) and (6). Here the quantity $\beta=[\rho(0)$ $\left.-\rho\left(C_{2}\right)\right] / \rho\left(C_{2}\right) C_{2} \equiv \delta / C_{2}$ is the molecular expansion coefficient. The parameter values are $g=980 \mathrm{~cm} / \mathrm{s}^{2}$ and $\delta=0.87 \times 10^{-4}$. We again adopt the uniform-density approximation, which ignores density changes except in the gravity term.

Equation (6) and the pressure term in Eq. (5) can be eliminated by introducing the stream function $\psi$,

$$
V_{y}=\frac{\partial \psi}{\partial z}, \quad V_{z}=-\frac{\partial \psi}{\partial y},
$$

and the vorticity $\omega$,

$$
\omega=\bar{\nabla}^{2} \psi .
$$

Combining with Eqs. (5), (21), and (20), we have

$$
\begin{gathered}
\frac{\partial \omega}{\partial t}=\frac{6}{5} \frac{\partial(\psi, \omega)}{\partial(y, z)}-\beta g \frac{\partial C}{\partial y}+\nu\left(\bar{\nabla}^{2}-\frac{12}{a^{2}}\right) \omega, \\
\frac{\partial C}{\partial t}=\frac{\partial(\psi, C)}{\partial(y, z)}+D_{C} \bar{\nabla}^{2} C-\alpha C\left(C-C_{2}\right)\left(C-C_{3}\right),
\end{gathered}
$$

where

$$
\frac{\partial\left(f_{1}, f_{2}\right)}{\partial(y, z)} \equiv \frac{\partial f_{1}}{\partial y} \frac{\partial f_{2}}{\partial z}-\frac{\partial f_{1}}{\partial z} \frac{\partial f_{2}}{\partial y} .
$$

We also require no-slip boundary conditions for the averaged fluid velocity and no-flow boundary conditions for the chemical concentration at the sidewalls.

To simulate the evolution of the autocatalytic reaction fronts in the narrow vertical slab, Eqs. (23), (24), and (25) are solved numerically using a rectangular mesh. The mesh size is varied to address the computational needs of each choice of $\mathcal{S}$ and $\Gamma$. The spatial derivatives are calculated using central differences; a five-point expansion is used to approximate the Laplacian. The time evolution is calculated using the explicit Euler method, and the Poisson equation is solved using a finite-term expansion method [15]. In this work, we used both four- and nine-term truncations. For $\Gamma<11$, the four-term truncation yields propagation speeds and front shapes that agree with the nine-term truncation. The results were checked using a cyclic reduction method [16] to solve the Poisson equation in test cases. The initial conditions consist of no fluid flow anywhere, together with small random perturbations in chemical concentration in the vicinity of a step-function concentration profile at the bottom of the mesh. The front propagates vertically in this bounded mesh, and reaches the upper boundary eventually. To simulate propagation in a vertically unbounded slab, the front is first allowed to travel a small distance and then is shifted back. At that time, unreacted fluid is added near the upper boundary, and reacted convectionless fluid is discarded near the lower boundary. This procedure is justified for vertical boundaries that are far away from the front.

\section{NUMERICAL SIMULATION RESULTS AND DISCUSSION}

The simulations employing the reaction-diffusion equation (Sec. IV) reveal that when the system is in the noconvection region (region I in Fig. 5), the front is flat and globally stable. Here the perturbations decay, leaving the steady flat profile described by Eq. (19), with the front speed differing from the theory by less than $1 \%$. The filled circles with error bars in Fig. 5 represent the marginal state for the onset of convection based on these simulations. The upper limit of the error bars represents the smallest value of $S \Gamma$ for which convection was observed, and the lower limit represents the largest value for which convection was not observed; the filled circles are located at the average of these two values. The thin-front approximation (Fig. 5, solid trace; see Sec. III) underestimates these values by about $15 \%$. This 

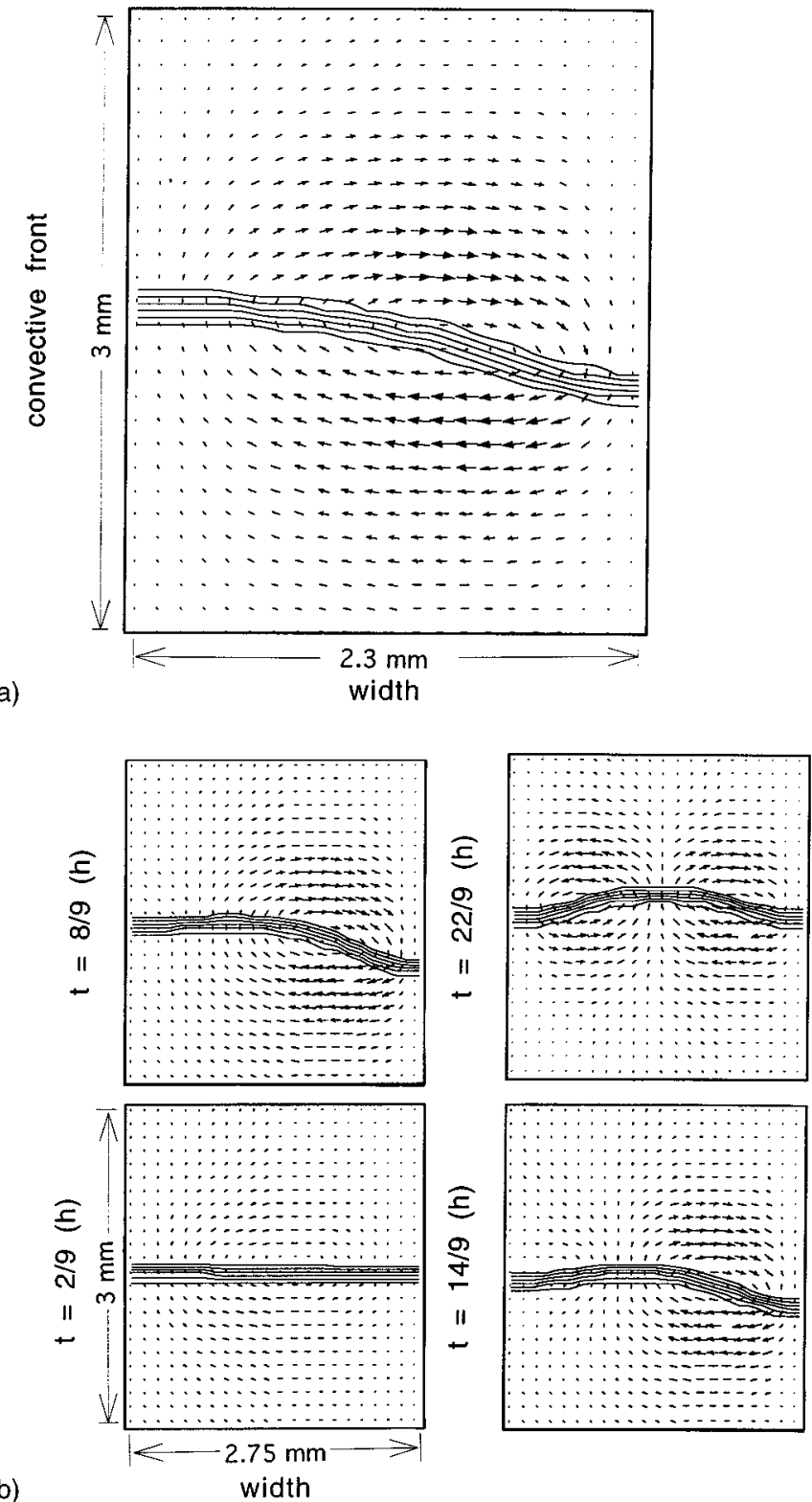

(b)

FIG. 6. (a) The steady one-roll state for $a=0.46 \mathrm{~mm}$ and $b=2.3 \mathrm{~mm}$. The solid lines are equiconcentration lines in the reaction front. These lines identify the reaction-front region where the concentration varies significantly, and thereby indicate the thickness of the reaction front. The simulation employed a grid space of 0.1 $\mathrm{mm}$ in the propagation direction and a time step of $1.0 \times 10^{-3} \mathrm{~s}$, and was carried out in a mesh $100 \times 25$ (only a small portion near the front is shown here). (b) Demonstration of the development from the one-roll state to the two-roll state for $a=0.55 \mathrm{~mm}$ and $b=2.75 \mathrm{~mm}$. The evolution of the front is represented at four stages.

is also true for the laterally unbounded system [14], where the thin-front critical wave number $q_{c}=48.74 \mathrm{~cm}^{-1}$ exceeds the finite-front critical wave number $q_{c}=46.39 \mathrm{~cm}^{-1}$ by $5 \%$, which corresponds to underestimates of the critical driving parameter $S \sim q_{c}^{-3}$ by $15 \%$.

Just above the marginal state (region II in Fig. 5), one convective roll grows in the vicinity of the front and eventually saturates, with a corresponding half-wavelength deformation of the formerly flat front [see Fig. 6(a)]. Near mar-

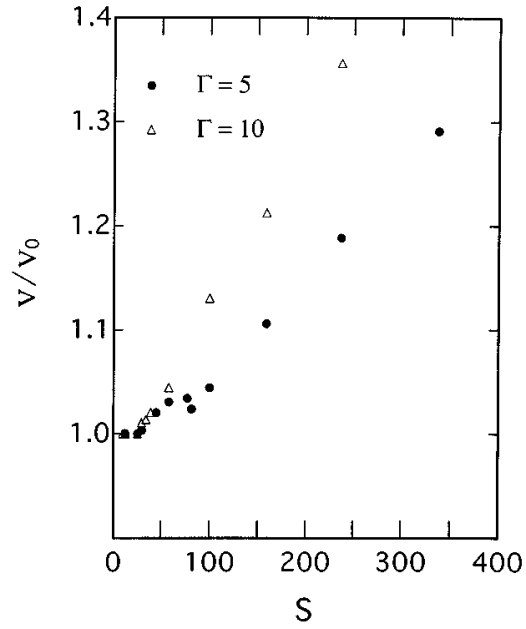

FIG. 7. The enhancement to the propagation speed $V$ of ascending fronts due to the onset of convection. The parameter $V_{0}$ is the speed of the flat front in the absence of convection. The cusp in $V$ (at $S \approx 80$ for $\Gamma=5$ ) reflects the transition from the one-roll state to the two-roll state.

ginal stability, the small values of the growth rate $\sigma$ demand a long time for the roll to grow and to saturate.

Our simulation reveals the existence of a transition from the steady one-roll state to a steady symmetric two-roll state (region III in Fig. 5). The open circles with error bars in Fig. 5 locate the transition. The upper limit of the error bars represents the smallest value of $S \Gamma$ for which the steady tworoll state was observed, and the lower limit represents the largest value for which the steady one-roll state was observed. Just above the transition, for initial conditions consisting of a slightly perturbed flat front, the one-roll state dominates initially owing to its largest growth rate [see Fig. 6(b)]. After a period of time depending on both the driving parameter $S$ and the aspect ratio $\Gamma$, the two-roll state emerges and eventually saturates, indicating that the one-roll state is nonlinearly unstable to the two-roll state. The larger the driving parameter $S$, the faster the two-roll state develops. The two-roll state corresponds to a full-wavelength deformation of the front [Fig. 6(b) at $t=22 / 9$ ].

Convection enhances the propagation speed $v$ of the fronts. Numerical data reveal that the increase in $v$ depends on both the driving parameter $S$ and the aspect ratio $\Gamma$ (Fig. 7 ). The cusp in $v$ (at $S \approx 80$ for $\Gamma=5$ ) reflects the transition to two rolls discussed above.

Simulations well above the transition to the two-roll state reveal edge suppression and a tertiary instability that breaks the symmetry of the two-roll state. These simulations were carried out for $S=463.4$ and $\Gamma=10$, which correspond to $a=1 \mathrm{~mm}$ and $b=10 \mathrm{~mm}$. Figure 5 ensures that the corresponding value $S \Gamma=4634$ greatly exceeds the marginal value $S \Gamma \approx 300$ for the transition to the two-roll state. Figure 8 shows the velocity fields for these simulations at four different stages during the development of the front. The small, random perturbations about the initially flat convectionless front lead to a first stage consisting of five rolls. This result follows approximation from the linear stability analysis for the unbounded system, which predicts a wavelength 


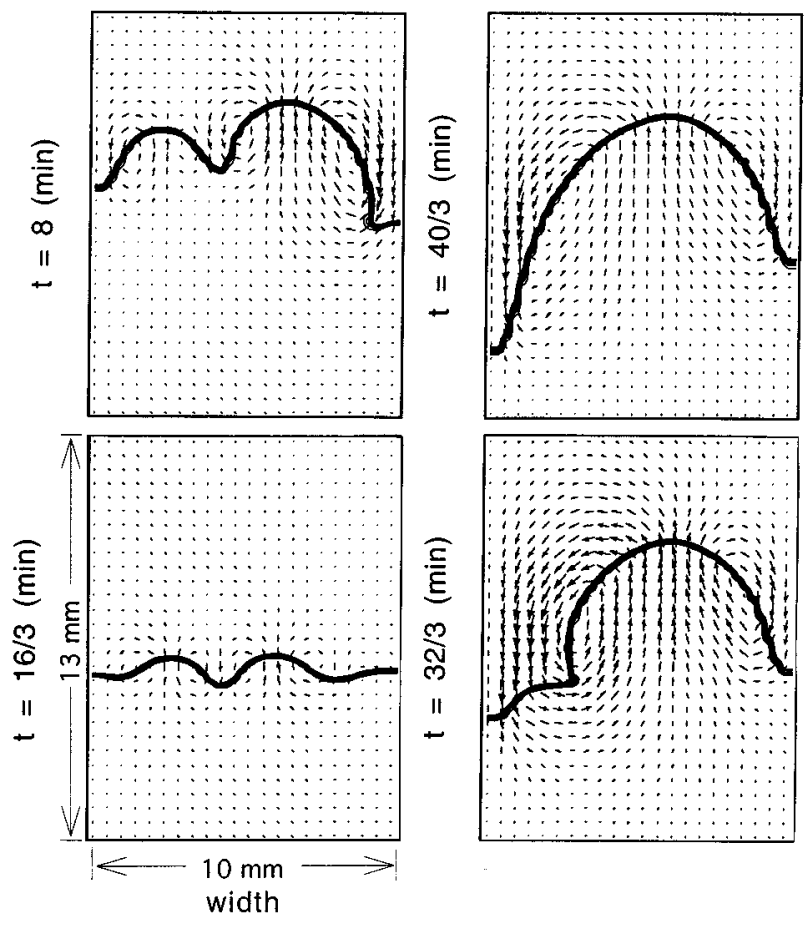

FIG. 8. Edge suppression for $a=1 \mathrm{~mm}$ and $b=10 \mathrm{~mm}$. The nonsymmetric two-roll state is evident in the final stage.

$\lambda_{m}=2 \pi a / q_{m}=3.55 \mathrm{~mm}$ for the fastest-growing mode [from Eq. (16)], and a corresponding number $b /\left(\lambda_{m} / 2\right) \approx 5.6$ of rolls (since each roll occupies half a wavelength). In the second and third stages, the nonlinear interactions between these five rolls lead to strong downward flows near the sidewalls as rolls are annihilated there. These strong flows temporarily suppress the propagation of the front at the edges of the front. Such edge suppression has been observed in experiments [17]. The fourth stage shows the final saturated state consisting of two unsymmetric rolls. This state indicates a tertiary transition from two symmetric rolls to two unsymmetric rolls above the transition from one to two symmetric rolls [see Figs. 5 and 6(b)]. The nature and location of this transition deserve further study.

Simulations well beyond the marginal condition also reveal fingering in the front. Our simulations of the evolution of fronts for $S=463.4$ and $\Gamma=3,5,10,14,17$, and 18 clearly demonstrate the formation, annihilation, and development of fingers in the fronts (see Fig. 9), indicating that the nonlinear dynamics mainly dominates the evolution of the fronts. The measured typical wavelength $\lambda$ that emerges initially is in the range $3.4-4.6 \mathrm{~mm}$. The average wavelength of $4.0 \mathrm{~mm}$ differs with the predicted maximum growth wavelength $\lambda_{m}=3.55 \mathrm{~mm}$ by $11 \%$. This difference could be due to the thin-front approximation.

Experiments designed to test the instability of ascending reaction fronts in a slab can be carried out by varying any of the parameters in $S=\delta g a^{3} / \nu D_{C}$, notably the density jump $\delta$ (through the various chemical concentrations) or the slab gap $a$. Varying the gap $a$ is more effective since $S$ is proportional to $a^{3}$. Decreasing $S$ will eventually yield a flat front for any given aspect ratio $\Gamma$. Note that whereas Fig. 5 is universal, the critical gap $a$ and width $b$ of the slab depend

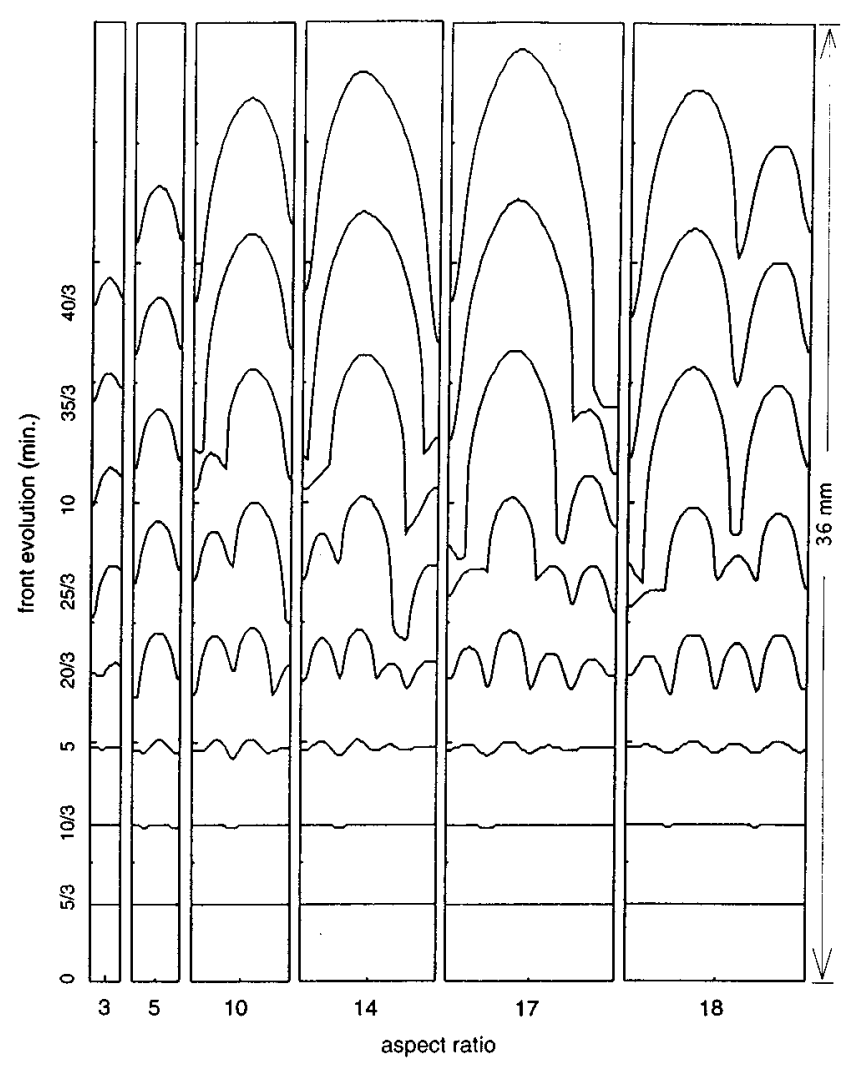

FIG. 9. Fingering in the fronts for different aspect ratios $\Gamma=3,5,10,14,17$, and 18 , with a fixed gap $a=1 \mathrm{~mm}$.

on the type of the reaction, the initial chemical concentrations, the fluid viscosity, etc. For example, for the iodatearsenous-acid reaction, Eq. (1) yields $S=463.4 a^{3}$ with $a$ measured in millimeters. For $\Gamma=6$, Fig. 5 yields the critical condition $S_{c} \Gamma=105$, which yields $a_{c}=0.34 \mathrm{~mm}$ and $b=2.04 \mathrm{~mm}$.

In conclusion, our nonlinear numerical simulation of the evolution of autocatalytic reaction fronts ascending a narrow vertical slab confirms our linear marginal stability analysis, and also reveals transitions to two-roll states, edge suppression, and fingering. An amplitude-equation analysis and careful experiments might shed further light on the transition to the two-roll state. The relation between initial perturbations and patterns developed in the fronts is also an interesting subject. Theoretically, we can study the relation through selected perturbations to start the numerical simulation. Experimentally, this might be realized by initiating the chemical reaction with controlled initial perturbations (through various electrode shapes or by initiating the reaction at selected points).

\section{ACKNOWLEDGMENTS}

We are indebted to Yunqing $\mathrm{Wu}$ for many valuable suggestions on the numerical work and for sharing his numerical code with us. Discussions with Eugenia Kuo, Stephen Morris, Desiderio Vasquez, and Joseph Wilder are gratefully acknowledged. This work was supported in part by National Science Foundation Grant No. RII-8922106 and the National Research Center for Coal and Energy. 
[1] J. A. Pojman, I. R. Epstein, T. J. McManus, and K. Showalter, J. Phys. Chem. 95, 1299 (1991).

[2] B. F. Edwards, J. W. Wilder, and K. Showalter, Phys. Rev. A 43, 749 (1991).

[3] A. Saul and K. Showalter, in Oscillations and Traveling Waves in Chemical System, edited by R. J. Field and M. Burger (Wiley, New York, 1985), p. 419; A. Hanna, A. Saul, and K. Showalter, J. Am. Chem. Soc. 104, 3838 (1982).

[4] D. A. Vasquez, B. F. Edwards, and J. W. Wilder, Phys. Rev. A 43, 6694 (1991).

[5] J. P. Keener and J. J. Tyson, Physica D 32, 307 (1988).

[6] J. Huang, D. A. Vasquez, B. F. Edwards, and P. Kolodner, Phys. Rev. E 48, 4378 (1993).

[7] H. Lamb, Hydrodynamics, 6th ed. (Cambridge University Press, Cambridge, 1932), Sec. 330.

[8] P. G. Saffman and G. I. Taylor, Proc. R. Soc. London Ser. A 245, 312 (1958); D. Bensimon, L. P. Kadanoff, S. Liang, B. I. Shraiman, and C. Tang, Rev. Mod. Phys. 58, 977 (1986).

[9] L. L. Green and T. D. Foster, J. Fluid Mech. 71, 675 (1975).
[10] H. C. Brinkman, Appl. Sci. Res. A 1, 27 (1947).

[11] A. A. Predtechensky, W. D. McCormick, J. B. Swift, A. G. Rossberg, and H. L. Swinney, Phys. Fluids 6, 3923 (1994).

[12] A. L. Fetter and J. D. Walecka, Theoretical Mechanics of Particles and Continua (McGraw-Hill, New York, 1980), Chap. 12.

[13] J. Boussinesq, Théorique Analytique de la Chaleur (GauthierVillars, Paris, 1903), Vol. 2; A. Oberbeck, Ann. Phys. Chem. (Leipzig) 7, 271 (1879); A. Oberbeck, Sitz. Ber. K. Preuss. Akad. Wiss. 383 and 1129 (1888).

[14] D. A. Vasquez, J. W. Wilder, and B. F. Edwards, J. Chem. Phys. 98, 2138 (1993).

[15] D. A. Vasquez, J. M. Littley, J. W. Wilder, and B. F. Edwards, Phys. Rev. E 50, 280 (1994); Y. Wu, D. A. Vasquez, B. F. Edwards, and J. W. Wilder, ibid. 51, 1119 (1995).

[16] R. Sweet, Siam J. Numer. Anal. 14, 706 (1977).

[17] M. R. Carey, S. W. Morris, and P. R. Kolodner, Phys. Rev. E 53, 6012 (1996). 